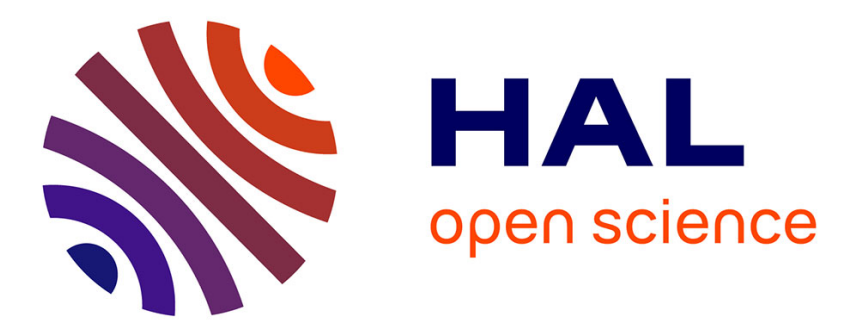

\title{
Enhancement of the electro-optical properties in hybrid organic-inorganic crystals by molecular engineering
} Jean-Paul Salvestrini, Mustapha Abarkan, Julien Zaccaro, Alain Ibanez

\section{To cite this version:}

Jean-Paul Salvestrini, Mustapha Abarkan, Julien Zaccaro, Alain Ibanez. Enhancement of the electrooptical properties in hybrid organic-inorganic crystals by molecular engineering. Journal of the Optical Society of America B, 2003, 20 (8), pp.1661-1665. 10.1364/JOSAB.20.001661 . hal-00186024

\section{HAL Id: hal-00186024 \\ https://hal.science/hal-00186024}

Submitted on 12 Jan 2022

HAL is a multi-disciplinary open access archive for the deposit and dissemination of scientific research documents, whether they are published or not. The documents may come from teaching and research institutions in France or abroad, or from public or private research centers.
L'archive ouverte pluridisciplinaire HAL, est destinée au dépôt et à la diffusion de documents scientifiques de niveau recherche, publiés ou non, émanant des établissements d'enseignement et de recherche français ou étrangers, des laboratoires publics ou privés.

\section{다)(1) $(5$}

Distributed under a Creative Commons Attribution - NonCommercial| 4.0 International 


\title{
Enhancement of the electro-optic properties in hybrid organic-inorganic crystals by molecular engineering
}

\author{
Jean Paul Salvestrini and Mustapha Abarkan \\ Laboratoire Matériaux Optiques, Photonique et Systèmes, Centre National de la Recherche Scientifique FRE 2304, \\ Université de Metz et Supelec, 2 rue E. Belin, 57070 Metz, France \\ Julien Zaccaro and Alain Ibanez \\ Laboratoire de Cristallographie, Centre National de la Recherche Scientifique, UPR 5031, Université J. Fourier et \\ Institut National Polytechnique de Grenoble, BP 166, 38042 Grenoble Cedex 09, France
}

\begin{abstract}
The electro-optic properties and their frequency dispersion of the 2-amino-5-nitropyridium monohydrogen L-tartrate (2A5NPLT) are investigated at room temperature at a wavelength of $632.8 \mathrm{~nm}$. The results show a large increase (more than three times higher) of the electro-optic coefficient values in the 2A5NPLT crystal compared with the 2A5NPDP crystal, which is one of the first crystals engineered with the 2-amino-5-nitropyridine highly polarizable chromophore.
\end{abstract}

\section{INTRODUCTION}

In the past two decades, extensive research has shown that organic crystals can exhibit nonlinear and thus electro-optic (EO) properties that are comparable with or even better than those of the best inorganic materials. ${ }^{1,2}$ This is due to highly polarizable molecules that involve a conjugated system of $\pi$ electrons such as polyenes or aromatic compounds. Nevertheless, because of their poor chemical stability and low thermal and mechanical resistance, the use of organic molecular crystals is currently limited in their industrial application.

To overcome these difficulties, a strategy has been developed that aims to build cohesive acentric crystalline structures based on host-guest chemistry. ${ }^{3}$ The attachment of highly polarizable organic cations onto various anionic matrices leads to short and three-dimensional (3-D) networks of short hydrogen bonds. Several polarizable molecules were selected in this crystal engineering and particularly 2-amino-5-nitropyridine (2A5NP). A great number of hydrogen bond salts having a 3-D acentric structure based on polar herringbone motifs were obtained with this push-pull molecule such as dihydrogen phosphate $^{4}$ or dihydrogen arsenate. ${ }^{5}$ These salts possess enhanced stability (chemical, thermal, mechanical) compared with corresponding molecular organic crystals. Moreover, they exhibit a wider transparency range and bulky crystal morphology. 6,7

Unfortunately, as shown recently, ${ }^{8,9}$ the EO properties of the first two compounds did not reach our expectation. Indeed, the larger value of the effective EO coefficient that we measured in the 2-amino-5-nitropyridine dihydrogen phosphate (2A5NPDP) crystal was equal to 11 $\mathrm{pm} / \mathrm{V}$. This value is of the same order of magnitude as that of the inorganic KDP family type. ${ }^{10}$ As has been shown in Refs. 8 and 9, the EO properties of the hybrid organic-inorganic crystals can be attributed mainly to organic cations (the contribution of the inorganic subnetwork to the EO coefficient can be neglected). Thus, we believe that the low EO coefficient value for these crystals arises from the misalignment between the charge transfer axis of the organic chromophores in the crystal and the light polarization direction. Indeed, the herringbone motif, which is favorable to second-harmonic generation, corresponds to a quite significant misalignment angle (35 deg in the case of 2 A5NPDP crystal) and thus yields to low EO properties.

From the crystal engineering mentioned above, a new type of hydrogen bond salt structure was discovered. ${ }^{11,12}$ This new material, 2-amino-5-nitropyridinium monohydrogen L-tartrate (2A5NPLT), was shown to have $2 \mathrm{~A} 5 \mathrm{NP}^{+}$cations nearly aligned with the crystallographic $b$ axis (see Fig. 1). In the following we propose to characterize the EO properties of this compound to check if this quite good alignment of the dipole moments yields an enhancement of the EO properties.

\section{PREPARATION OF 2A5NPLT CRYSTAL AND SAMPLE}

High-purity 2A5NPLT salt was synthesized from the dissolution of purified 2A5NP (multiple sublimation) and L-Tartaric acid in hot acetic acid aqueous solution (14 M). Crystals were grown from solution both by the standard temperature-lowering method ${ }^{13}$ and a rapid growth technique derived from the one developed for $\mathrm{KH}_{2} \mathrm{PO}_{4} \cdot{ }^{14,15}$ With the latter technique, we obtained single crystals, 


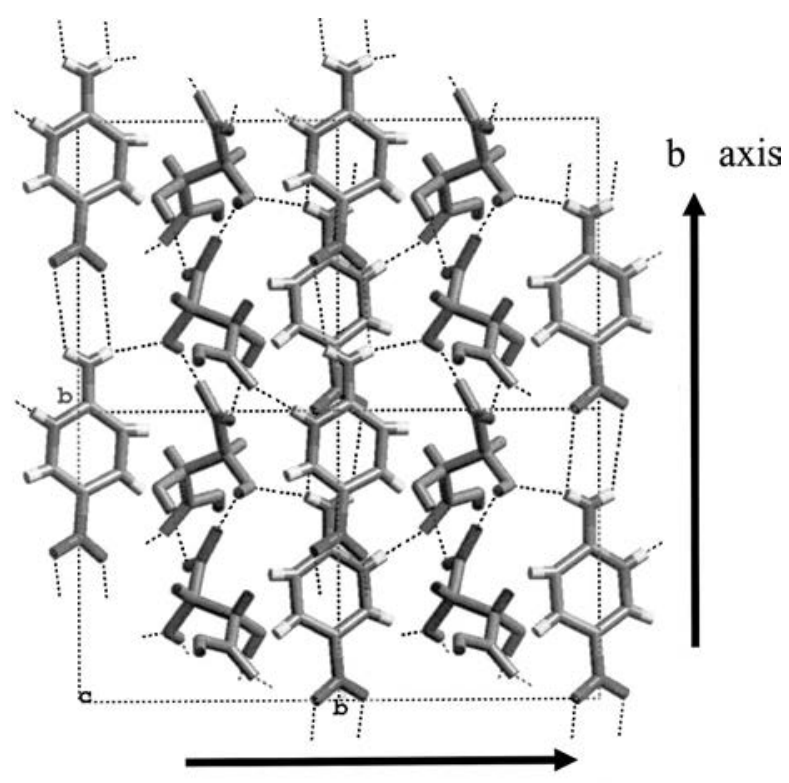

a axis

Fig. 1. Projection of the polar structure of 2A5NPLT in the $a b$ plane showing a quasi-perfect alignment of the 2-amino-5nitropyridium cations.

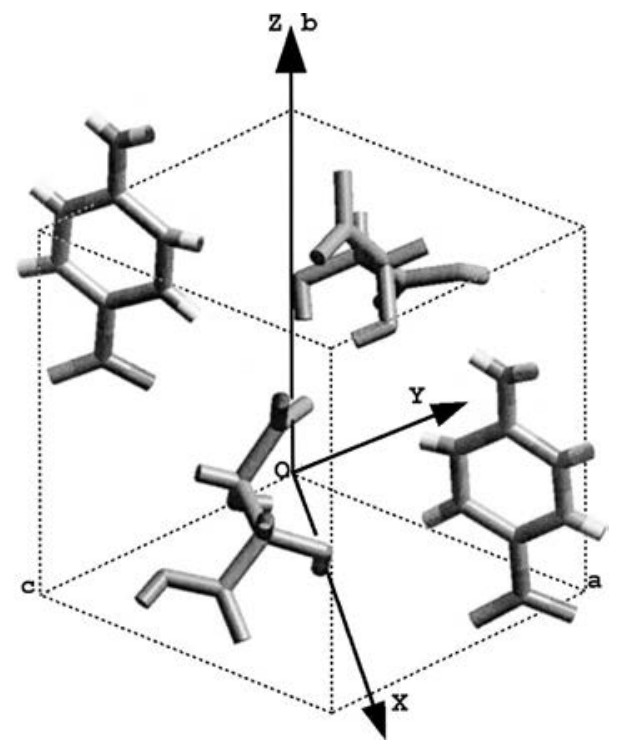

Fig. 2. Orientation of dielectric and crystallographic axes with respect to each other in 2A5NPLT crystals. The dielectric $X$ axis is in the $(a c)$ plane at $56^{\circ}$ from the crystallographic $c$ axis and the $Y$ axis lies in the $2 \mathrm{~A}_{5} \mathrm{NP}^{+}$molecular plane.

several millimeters in size (typically $5 \mathrm{~mm} \times 5 \mathrm{~mm} \times 10$ $\mathrm{mm}$ ), in approximately 3 weeks. The quality of these crystals was controlled by x-ray diffraction topography. The arrangement of the optically active molecule in the structure is highly polar, the charge transfer axis of the $2 \mathrm{~A} \mathrm{NP}^{+}$cation being almost aligned $\left(3.6^{\circ}\right)$ with the dielectric $Z$ axis (corresponding to the crystallographic $b$ axis, as seen in Fig. 2). According to Ref. 11, the dielectric $X$ axis is in the $(a c)$ plane at $56^{\circ}$ from the crystallographic $c$ axis and the $Y$ axis lies in the $2 \mathrm{~A}_{5} \mathrm{NP}^{+}$molecular plane. As the 2A5NPLT crystals belongs to the $P 2_{1}$ symmetry group, where the twofold axis is along the prin- cipal dielectric $Z$ axis, the linear EO properties of this crystal are described in abbreviated notation by eight coefficients, $r_{13}, r_{23}, r_{33}, r_{63}, r_{42}, r_{52}, r_{41}$, and $r_{51}$. Here we focus our attention only on coefficients $r_{13}, r_{23}$, and $r_{33}$ for which the contribution of the organic molecule to the EO properties is the largest. ${ }^{9}$ If the light beam propagates along the principal $X$ axis, the principal $Y$ axis or the $Z$ axis and the electrical field is applied along the $Z$ axis, the field-induced birefringences in $(Y, Z),(X, Z)$, and $(X, Y)$ planes are given, respectively, by

$$
\begin{aligned}
& \Delta n_{y z}(E)=-\frac{n_{2}{ }^{3} r_{a} E_{z}}{2}, \quad \Delta n_{x z}(E)=-\frac{n_{1}{ }^{3} r_{b} E_{z}}{2}, \\
& \Delta n_{x y}(E)=-\frac{n_{1}{ }^{3} r_{c} E_{z}}{2}
\end{aligned}
$$

where the effective coefficients $r_{a}, r_{b}$, and $r_{c}$ are defined by

$$
\begin{aligned}
& r_{a}=r_{23}-\frac{n_{3}^{3}}{n_{2}{ }^{3}} r_{33}, \quad r_{b}=r_{13}-\frac{n_{3}^{3}}{n_{1}^{3}} r_{33}, \\
& r_{c}=r_{13}-\frac{n_{2}{ }^{3}}{n_{1}{ }^{3}} r_{23} .
\end{aligned}
$$

Finally, the field-induced phase shift between the two components of the light polarization can be expressed by

$$
\Gamma(E)=\frac{2 \pi L}{\lambda} \Delta n(E),
$$

where $L$ is the crystal length along the light propagation direction, $\lambda$ is the wavelength of the light beam, and $\Delta n(E)$ is the field-induced birefringence in the light path.

Only one sample is necessary for the measurement of EO coefficients $r_{a}, r_{b}$, and $r_{c}$. The sample was prepared as a parallelepiped with the principal axes $X, Y$, and $Z$ perpendicular to the faces. The six faces were optically polished. Gold electrodes were evaporated on the $Z$ faces (electric field should be applied along the $Z$ axis) taking care to let a small surface free of gold (see Fig. 3) to allow

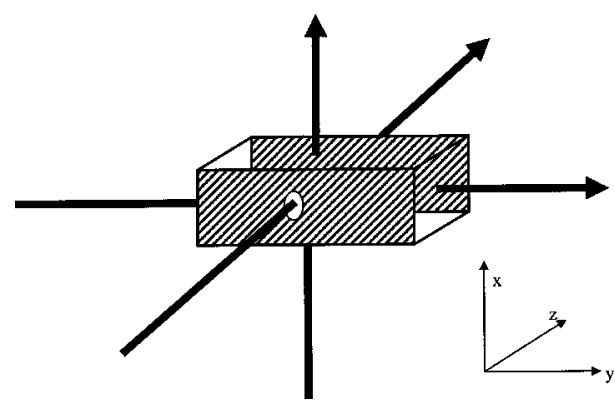

Fig. 3. Schema of the prepared sample used for the EO characterization of the 2A5NPLT crystals. Gold electrodes were deposited on $z$ faces taking care to let a small surface free of gold to allow the light beam to go through the sample in the $Z$ direction. This sample allows the measurement of the EO coefficient $r_{a}$, $r_{b}$, and $r_{c}$ for a light beam that propagates along the $X, Y$, and $Z$ directions, respectively, and for an applied electric field along the $Z$ axis. 
the light beam to go through the sample in the $Z$ direction and thus to have the possibility to measure the EO coefficient $r_{c}$.

\section{EXPERIMENTAL SETUP AND TECHNIQUE}

To determine the EO coefficients $r_{a}$ and $r_{b}$ and their frequency dependence, we used the time response method $(\mathrm{TRM})^{16,17}$ with the Sénarmont setup, which allows us to obtain the clamped $\left(r^{S}\right)$ and unclamped $\left(r^{T}\right)$ EO coefficients corresponding, respectively, to the measured coefficients at short (or high-frequency) and long (or lowfrequency) periods of time. The difference between the values of $r^{T}$ and $r^{S}$ gives the acoustic contribution to the EO coefficient. This contribution arises from indirect modulation of the susceptibility by means of crystal deformations ${ }^{18}$ and can be written as

$$
r_{i j k}^{T}-r_{i j k}^{S}=\sum_{m, n} p_{i j m n} d_{m n k},
$$

where $p_{i j m n}$ and $d_{m n k}$ are the components of the elastooptic and piezoelectric tensor, respectively.

The method that we used consists in the measurement of the time response of the EO crystal to a voltage step. We obtained the latter by switching a large voltage (up to $1 \mathrm{kV}$ ) in a short period of time (a few nanoseconds). At the point of the transmittance curve of the Sénarmont setup corresponding to the maximum of modulation intensity, the time dependence of the variation of the transmitted beam intensity $\Delta i(t)$ induced by the applied voltage $\Delta V(t)$ yields the time response of the EO crystal under test as

$$
\Delta i(t)=\frac{\pi n_{\mathrm{eff}}^{3} L I_{0}}{2 \lambda d} r_{\mathrm{eff}}(t) \otimes \Delta V(t),
$$

where $\otimes$ is the convolution operator and $r_{\text {eff }}(t)$ is the actual value of the $\mathrm{EO}$ coefficient. $I_{0}=I_{\max }-I_{\min }$ represents the total intensity range of the transfer function and $d$ is the interelectrode spacing. From the measurement of $\Delta i(t)$ and $\Delta V(t)$, the frequency dispersion of the EO coefficients can be derived from the ratio of $\Delta i(v)$ and $\Delta V(v)$ which were obtained by the $Z$ transform of signals $\Delta i(t)$ and $\Delta V(t)$, respectively ${ }^{15}$ :

$$
r_{\mathrm{eff}}(\nu)=\frac{2 \lambda d}{\pi n_{\mathrm{eff}}^{3} I_{0} L} \frac{\Delta i(\nu)}{\Delta V(\nu)} .
$$

We also determined the coefficient $r_{c}$ using the Sénarmont setup by means of the ac modulation method. ${ }^{19}$ Within the TRM, the efficiency of the EO effect in this configuration [the ratio $L / d$ in Eq. (5) is equal to 1 in this case, leading to a weak induced phase shift $\Gamma$ ] was indeed not enough to reach a good signal-to-noise ratio.

We have measured the time response and determined the frequency dispersion of the $r_{a}$ and $r_{b}$ coefficients. We also measured the value of the $r_{c}$ coefficient for frequencies of the applied electric field that vary between 1 and $10 \mathrm{kHz}$. We obtained all these data using the 632.8-nm wavelength of a He-Ne laser. The amplitudes of the ap- plied voltage that we used were in the $250-500-\mathrm{V}$ range. The dimensions of the sample were $X \times Y \times Z=5 \mathrm{~mm}$ $\times 5 \mathrm{~mm} \times 1.1 \mathrm{~mm}$.

\section{RESULTS AND DISCUSSION}

As an example, Fig. 4 displays a typical record of applied voltage and corresponding induced optical intensity variation versus time obtained during EO measurements when we used the TRM. It corresponds to the case of the $r_{a}$ coefficient. In the long-time range the optical signal oscillates with a period that corresponds to the main piezoelectric frequency resonance. ${ }^{16}$ The oscillations of the optical signal tend to vanish for a time longer than several tens of microseconds. It is to be noted that these oscillations do not exist in the short-time regime $(<100 \mathrm{~ns})$ since acoustic waves need more time to go through the crystal. In this time range the optical response is flat (see the inset in Fig. 4). The relatively weak amplitude of the oscillations and the relatively small difference between the value of the optical signal at long and short times indicate that the acoustic contribution to the EO effect in this material is not strong.

The frequency dispersion of the EO coefficient $r_{a}$ can be derived, according to Eq. (6), from the ratio of $\Delta i(\nu)$ and $\Delta V(\nu)$, which were obtained by the $Z$ transform of the $\Delta i(t)$ and $\Delta V(t)$ signals shown in Fig. 4. This mathematical treatment was carried out for both EO coefficients $r_{a}$ and $r_{b}$, and the results are plotted in Fig. 5 . The frequency varies in the range from $10 \mathrm{kHz}$ to 200 $\mathrm{MHz}$, limited in the low-frequency part by the duration of the voltage pulse and in the high-frequency part by the rise time of the voltage step. On both sides of the acoustic resonances, the dispersion of the EO coefficients $r_{a}$ and $r_{b}$ is flat and the corresponding value of each plateau allows us to determine the unclamped (low-frequency) and clamped (high-frequency) values of the coefficients.

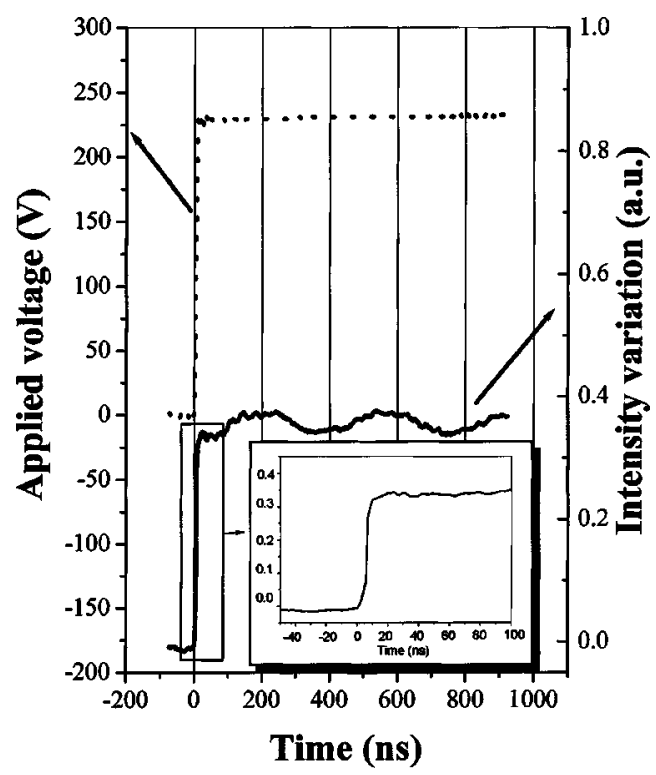

Fig. 4. Typical record of applied voltage (left part) and corresponding induced intensity variation (right part) versus time obtained during EO measurements when we used the TRM, corresponding to the case of coefficient $r_{a}$ in the 2A5NPLT crystal. 

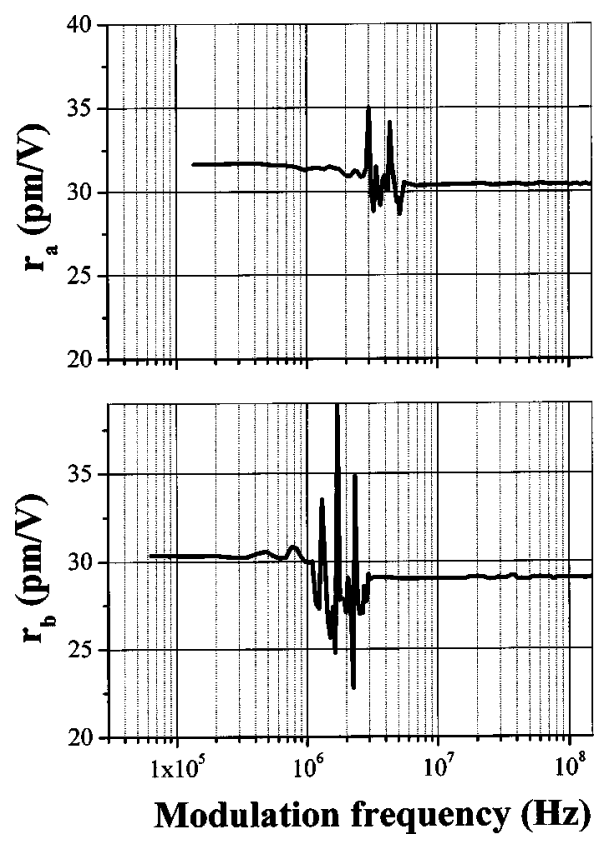

Fig. 5. Frequency dispersion of EO coefficients $r_{a}$ and $r_{b}$ deduced from time response measurements.

Table 1. Measured Values of the Unclamped $\left(r^{T}\right)$ and Clamped $\left(r^{S}\right)$ Effective EO Coefficients $r_{a}, r_{b}$, and $r_{c}$ in 2A5NPLT at $632.8 \mathrm{~nm}$

\begin{tabular}{cccc}
\hline Coefficient & $r^{T}(\mathrm{pm} / \mathrm{V})$ & $r^{S}(\mathrm{pm} / \mathrm{V})$ & $\begin{array}{c}\text { Acoustic Contributions } \\
{ }^{a}\end{array}$ \\
\hline$r_{a}$ & $32 \pm 1.5$ & $30.5 \pm 1.2$ & 4.5 \\
$r_{b}$ & $30.3 \pm 1.2$ & $29 \pm 1.1$ & 4.5 \\
$r_{c}$ & $10 \pm 0.5$ & - & - \\
\hline
\end{tabular}

${ }^{a}$ The last column lists the relative acoustic contribution to the EO coefficient and is defined by $\left(r^{T}-r^{S} / r^{T}\right)$.

Table 2. Comparison of the Low-Frequency EO Coefficients $r_{a}, r_{b}$, and $r_{c}$ and of the Acoustic Contributions in 2A5NPLT and 2A5NPDP

\begin{tabular}{lcc}
\hline \multicolumn{1}{c}{ Coefficient } & 2A5NPLT & 2A5NPDP \\
\hline$r_{a}{ }^{T}$ & $32 \pm 1.5$ & $11 \pm 1.2$ \\
$r_{b}{ }^{T}$ & $30.3 \pm 1.2$ & $9.5 \pm 1$ \\
$r_{c}{ }^{T}$ & $10 \pm 0.5$ & $0.3 \pm 0.04$ \\
$\left(r_{a}{ }^{T}-r_{a}{ }^{S}\right) / r_{a}{ }^{T}$ & $4.5 \%$ & $9 \%$ \\
\hline
\end{tabular}

All these values for $r_{a}$ and $r_{b}$ are reported in Table 1 as well as the low-frequency $(10-\mathrm{kHz})$ value of coefficient $r_{c}$, which was measured with the ac modulation technique. As mentioned above it is obvious that the difference between the high- and the low-frequency values of both coefficients $r_{a}$ and $r_{b}$ is weak leading to a less than 5\% acoustic contribution, which means that the EO effect in this compound originates essentially from electronic and ionic contributions.

The values of $r_{a}$ and $r_{b}$ are quite important since they are larger than those of lithium niobate, for example, $r_{a}$ $=r_{b}=20 \mathrm{pm} / \mathrm{V} \cdot{ }^{20}$ In contrast, the value of coefficient $r_{c}$ does not reach so large a value. This difference between $r_{a}$ and $r_{b}$ from one part and $r_{c}$ from another part in the 2A5NPLT crystal is comparable with that observed for the 2A5NPDP crystal. Table 2 depicts this comparison. The anisotropy of the EO coefficient values in 2A5NPLT could be also attributed, as in 2A5NPDP, to anisotropy of the organic chromophore in its contribution to the polarizability. ${ }^{9}$

\section{CONCLUSION}

We have measured the frequency dispersion of EO coefficients $r_{a}, r_{b}$, and $r_{c}$ of 2A5NPLT crystals. These results were compared with those of the 2A5NPDP crystal. We have observed a large increase of the values of the $\mathrm{EO}$ coefficients indicating a better alignment of the $2 \mathrm{~A}^{2} \mathrm{NP}^{+}$organic cations charge transfer axis in the 2A5NPLT crystal. The lattice deformation (giving rise to acoustic contribution in the EO properties) in 2A5NPLT is weak and even lower than those in 2A5NPDP. This could be explained by the denser 3-D hydrogen bond network in the 2A5NPLT crystal than in the 2A5NPDP crystal. We can guess, as we demonstrated in the case of 2A5NPDP crystal, ${ }^{9}$ that the magnitude of EO coefficients $r_{13}, r_{23}$, and $r_{33}$ is mainly electronic in origin, arising from the $\pi$ electron of the $2 \mathrm{~A}^{2} \mathrm{NP}^{+}$cations. Thus, contribution to the EO effect of the counter ions, tartrate anions for 2A5NPLT, and phosphates for 2A5NPDP is negligible for the EO properties.

The e-mail address for J.-P. Salvestrini is salvestr @ese-metz.fr.

\section{REFERENCES}

1. Ch. Bosshard, K. Sutter, R. Schlesser, and P. Günter, "Electro-optics effects in molecular crystals," J. Opt. Soc. Am. B 10, 867-885 (1993).

2. G. Knöpfle, Ch. Bosshard, R. Schlesser, and P. Günter, "Optical, nonlinear optical, and electrooptical properties of MNBA crystals," IEEE J. Quantum Electron. 30, 13031312 (1994).

3. R. Masse, M. Bagieu-Beucher, J. Pecaut, J. P. Levy, and J. Zyss, "Design of organic-inorganic polar crystals for quadratic nonlinear optics," Nonlinear Opt. 5, 413-423 (1993).

4. R. Masse and J. Zyss, "A new approach in the design of polar crystals for quadratic nonlinear optics exemplified by the synthesis of 2-amino-5-nitropyridium-dihydrogen monophosphate (2A5NPDP)," Mol. Eng. 1, 141-152 (1991).

5. J. Pecaut, Y. Lefur, and R. Masse, "Crystal engineering and structural investigations of the 2-amino-5-nitropyridium salts $\mathrm{C}_{5} \mathrm{H}_{6} \mathrm{~N}_{3} \mathrm{O}_{2}{ }^{+} \mathrm{HSO}_{4}{ }^{-}$and $\mathrm{C}_{5} \mathrm{H}_{6} \mathrm{~N}_{3} \mathrm{O}_{2}{ }^{+} \mathrm{H}_{2} \mathrm{AsO}_{4}{ }^{-}$," Acta Crystallogr., Sect. B 49, 535-541 (1993).

6. A. Ibanez, J. P. Levy, C. Mouget, and E. Prieur, "Crystal growth of a promising nonlinear optical material: 2-amino-5-nitropyridium-chloride," J. Solid State Chem. 129, 22-29 (1997).

7. J. Zaccaro, B. Capelle, and A. Ibanez, "Crystal growth of hybrid nonlinear optical materials: 2-amino-5nitropyridinium dihydrogenphosphate and dihydrogenarsenate," J. Cryst. Growth 180, 229-237 (1997).

8. J. P. Salvestrini, J. Zaccaro, A. Ibanez, and M. D. Fontana, "Investigation of electro-optic modulation from organicinorganic crystals," Appl. Phys. B 67, 761-763 (1998).

9. J. Zaccaro, J. P. Salvestrini, A. Ibanez, P. Ney, and M. D. Fontana, "Electric-field frequency dependence of Pockels co- 
efficients in 2-amino-5-nitropyridium dihydrogen phosphate organic-inorganic crystals," J. Opt. Soc. Am. B 17, 427-432 (2000).

10. A. Yariv and P. Yeh, Optical Waves in Crystals (Wiley, New York, 1984)

11. J. Zyss, R. Masse, M. Bagieu-Beucher, and J. P. Levy, "Quasi-perfect polar alignment of nonlinear chromophores in a crystalline H-bonded guest-host structure: 2-amino5-nitropyridinium-L-monohydrogentartrate," Adv. Mater. 5, 120-124 (1993).

12. O. Watanabe, T. Noritake, Y. Hirose, A. Okada, and T. Kurauchi, "Synthesis, crystal structure and nonlinear optical properties of 2-amino-5-nitropyridine-L-(+)-tartrate, a new second-harmonic-generation crystal," J. Mater. Chem. 3, 1053-1057 (1993).

13. J. Zaccaro, F. Lorut, and A. Ibanez, "Crystal growth of a stable nonlinear optical organic material: 2-amino-5nitropyridium monohydrogen L-tartrate," J. Mater. Chem. 9, 1091-1095 (1999).

14. N. P. Zaitseva, J. J. de Yoreo, M. R. Dehaven, R. L. Vital, K. E. Montgomery, R. Richardson, and L. J. Atherton, "Rapid growth of large-scale $(40-55 \mathrm{~cm}) \mathrm{KH}_{2} \mathrm{PO}_{4}$ crystals," J. Cryst. Growth 180, 255-262 (1997).

15. J. Zaccaro, J. Haertwig, J. Baruchel, and A. Ibanez, "Rapid growth of organic-inorganic hybrid single crystals," J Cryst. Growth 204, 325-332 (1999).

16. M. Abarkan, J. P. Salvestrini, M. Aillerie, and M. D. Fontana, "Frequency dispersion of electro-optical properties over a wide range by means of time response analysis," Appl. Opt. 42, 2346-2353 (2003).

17. R. Spreiter, Ch. Bosshard, F. Pan, and P. Günter, "High frequency response and acoustic phonon contribution of the linear electro-optic effect in DAST," Opt. Lett. 22, 564-566 (1997).

18. J. P. Salvestrini, M. D. Fontana, B. Wyncke, and F. Brehat, "Comparative measurements of the frequency dependence of electro-optical and dielectric coefficients in inorganic crystals," Nonlinear Opt. 17, 271-280 (1997).

19. L. Guilbert, J. P. Salvestrini, H. Hassan, and M. D. Fontana, "Combined effects due to phase, intensity, and contrast in electrooptic modulation: application to ferroelectric materials," IEEE J. Quantum Electron. 35, 273-281 (1999).

20. M. Aillerie, M. D. Fontana, F. Abdi, C. Carabatos-Nedelec, N. Theofanous, and G. Alexakis, "Influence of the temperature-dependent spontaneous birefringence in the electro-optic measurements of $\mathrm{LiNbO}_{3}$," J. Appl. Phys. 65, 2406-2408 (1989). 